\title{
Water distribution in shocked regions of the NGC 1333-IRAS 4A protostellar outflow ${ }^{\star}$
}

\author{
G. Santangelo ${ }^{1,2}$, B. Nisini ${ }^{2}$, C. Codella ${ }^{1}$, A. Lorenzani ${ }^{1}$, U. A. Yıldız ${ }^{3}$, S. Antoniucci ${ }^{2}$, P. Bjerkeli ${ }^{4,5,6}$, S. Cabrit ${ }^{7}$, \\ T. Giannini² ${ }^{2}$ L. E. Kristensen ${ }^{8}$, R. Liseau ${ }^{6}$, J. C. Mottram ${ }^{9}$, M. Tafalla ${ }^{10}$, and E. F. van Dishoeck ${ }^{9}, 11$ \\ 1 Osservatorio Astrofisico di Arcetri, Largo Enrico Fermi 5, 50125 Florence, Italy \\ e-mail: gina@arcetri.astro.it \\ 2 Osservatorio Astronomico di Roma, via di Frascati 33, 00040 Monteporzio Catone, Italy \\ 3 Jet Propulsion Laboratory, California Institute of Technology, 4800 Oak Grove Drive, Pasadena CA 91109, USA \\ 4 Niels Bohr Institute, University of Copenhagen, Juliane Maries Vej 30, 2100 Copenhagen Ø., Denmark \\ 5 Centre for Star and Planet Formation and Natural History Museum of Denmark, University of Copenhagen, Øster Voldgade 5-7, \\ 1350 Copenhagen K., Denmark \\ ${ }^{6}$ Department of Earth and Space Sciences, Chalmers University of Technology, Onsala Space Observatory, 43992 Onsala, Sweden \\ 7 LERMA, Observatoire de Paris, UMR 8112 of the CNRS, 61 Av. de l'Observatoire, 75014 Paris, France \\ 8 Harvard-Smithsonian Center for Astrophysics, 60 Garden Street, Cambridge MA 02138, USA \\ ${ }^{9}$ Leiden Observatory, Leiden University, PO Box 9513, 2300 RA Leiden, The Netherlands \\ 10 Observatorio Astronómico Nacional (IGN), Alfonso XII 3, 28014 Madrid, Spain \\ 11 Max Planck Institut für Extraterrestrische Physik (MPE), Giessenbachstr.1, 85748 Garching, Germany
}

Received 18 April 2014 / Accepted 19 June 2014

\section{ABSTRACT}

Context. Water is a key molecule in protostellar environments because its line emission is very sensitive to both the chemistry and the physical conditions of the gas. Observations of $\mathrm{H}_{2} \mathrm{O}$ line emission from low-mass protostars and their associated outflows performed with HIFI onboard the Herschel Space Observatory have highlighted the complexity of $\mathrm{H}_{2} \mathrm{O}$ line profiles, in which different kinematic components can be distinguished.

Aims. The goal is to study the spatial distribution of $\mathrm{H}_{2} \mathrm{O}$, in particular of the different kinematic components detected in $\mathrm{H}_{2} \mathrm{O}$ emission, at two bright shocked regions along IRAS 4A, one of the strongest $\mathrm{H}_{2} \mathrm{O}$ emitters among the Class 0 outflows.

Methods. We obtained Herschel-PACS maps of the IRAS 4A outflow and HIFI observations of two shocked positions. The largest HIFI beam of $38^{\prime \prime}$ at $557 \mathrm{GHz}$ was mapped in several key water lines with different upper energy levels, to reveal possible spatial variations of the line profiles. A large velocity gradient (LVG) analysis was performed to determine the excitation conditions of the gas.

Results. We detect four $\mathrm{H}_{2} \mathrm{O}$ lines and $\mathrm{CO}(16-15)$ at the two selected shocked positions. In addition, transitions from related outflow and envelope tracers are detected. Different gas components associated with the shock are identified in the $\mathrm{H}_{2} \mathrm{O}$ emission. In particular, at the head of the red lobe of the outflow, two distinct gas components with different excitation conditions are distinguished in the HIFI emission maps: a compact component, detected in the ground-state water lines, and a more extended one. Assuming that these two components correspond to two different temperature components observed in previous $\mathrm{H}_{2} \mathrm{O}$ and CO studies, the LVG analysis of the $\mathrm{H}_{2} \mathrm{O}$ emission suggests that the compact (about $3^{\prime \prime}$, corresponding to about $700 \mathrm{AU}$ ) component is associated with a hot $(T \sim 1000 \mathrm{~K})$ gas with densities $n_{\mathrm{H}_{2}} \sim(1-4) \times 10^{5} \mathrm{~cm}^{-3}$, whereas the extended $\left(10^{\prime \prime}-17^{\prime \prime}\right.$, corresponding to $\left.2400-4000 \mathrm{AU}\right)$ one traces a warm $(T \sim 300-500 \mathrm{~K})$ and dense gas $\left(n_{\mathrm{H}_{2}} \sim(3-5) \times 10^{7} \mathrm{~cm}^{-3}\right)$. Finally, using the CO (16-15) emission observed at $\mathrm{R} 2$ and assuming a typical $\mathrm{CO} / \mathrm{H}_{2}$ abundance of $10^{-4}$, we estimate the $\mathrm{H}_{2} \mathrm{O} / \mathrm{H}_{2}$ abundance of the warm and hot components to be $(7-10) \times 10^{-7}$ and $(3-7) \times 10^{-5}$.

Conclusions. Our data allowed us, for the first time, to resolve spatially the two temperature components previously observed with HIFI and PACS. We propose that the compact hot component may be associated with the jet that impacts the surrounding material, whereas the warm, dense, and extended component originates from the compression of the ambient gas by the propagating flow.

Key words. stars: low-mass - ISM: jets and outflows - ISM: molecules - ISM: individual objects: NGC 1333-IRAS 4A stars: formation

\section{Introduction}

Molecular outflows represent direct evidence of the earliest phases of star formation when collimated jets are driven (e.g., Arce et al. 2007; Ray et al. 2007). Shock fronts are generated

* PACS maps and HIFI spectra (FITS format) are only available at the CDS via anonymous ftp to

cdsarc.u-strasbg.fr (130.79.128.5) or via

http://cdsarc.u-strasbg.fr/viz-bin/qcat?]/A+A/568/A125 at the point of impact of the ejected material with the surrounding cloud, inducing changes in the chemical composition and enhancing the abundance of several species. Along with $\mathrm{H}_{2}$ and $\mathrm{CO}, \mathrm{H}_{2} \mathrm{O}$ is one of the main cooling agents in shocks (Kaufman \& Neufeld 1996; Flower \& Pineau des Forêts 2010; Karska et al. 2013). It is also very sensitive to physical conditions and chemical processes of the shocked gas (van Dishoeck et al. 2011). An increase of the water gas-phase abundance from $<10^{-7}$ up to $3 \times 10^{-4}$ is expected in warm shocked gas $(\gtrsim 100 \mathrm{~K})$ 
because of the combined effects of sputtering of ice mantles and high-temperature reactions that drive atomic oxygen into $\mathrm{H}_{2} \mathrm{O}$ (Hollenbach \& McKee 1989; Kaufman \& Neufeld 1996; Flower \& Pineau des Forêts 2010; Suutarinen et al. 2014).

Systematic observations of multiple $\mathrm{H}_{2} \mathrm{O}$ transitions in prototypical star-forming regions have been conducted with Herschel (Pilbratt et al. 2010). Thanks to its much higher sensitivity and spectral resolution and its smaller beam size with respect to previous space missions, such as ISO, Odin, and SWAS, Herschel has provided strong constraints on the water abundance and physical conditions in water-emitting gas. In particular, the Water In Star-forming regions with Herschel (WISH, van Dishoeck et al. 2011) key program has been dedicated to the study of the physical and dynamical properties of water and its role in shock chemistry. The $\mathrm{H}_{2} \mathrm{O}$ line profiles observed with the Heterodyne Instrument for the Far Infrared (HIFI, de Graauw et al. 2010) at outflow shocks show several kinematic components along with variations with excitation energy (Kristensen et al. 2012; Vasta et al. 2012; Santangelo et al. 2012). The observed $\mathrm{H}_{2} \mathrm{O}$ emission probes warm $(>200 \mathrm{~K})$ and very dense gas $\left(n_{\mathrm{H}_{2}} \gtrsim 10^{6} \mathrm{~cm}^{-3}\right)$, which is associated with high- $J \mathrm{CO}$ emission (e.g Karska et al. 2013; Santangelo et al. 2013) and is not traced by other molecules seen from the ground, such as low- $J$ $\mathrm{CO}$ and $\mathrm{SiO}$ (e.g., Vasta et al. 2012; Nisini et al. 2013; Tafalla et al. 2013; Santangelo et al. 2013). The differences in line profiles of the various tracers also confirm the uniqueness of $\mathrm{H}_{2} \mathrm{O}$ as a probe of shocked gas.

A two-temperature-components model has been suggested by Santangelo et al. (2013) to reproduce the $\mathrm{H}_{2} \mathrm{O}, \mathrm{CO}$, and mid-IR $\mathrm{H}_{2}$ lines, observed along the L1448 and L1157 protostellar outflows. This model consists of 1) an extended warm component $(T \sim 500 \mathrm{~K})$ traced by lower excitation $\mathrm{H}_{2} \mathrm{O}$ emission $\left(E_{\mathrm{u}} \lesssim 140 \mathrm{~K}\right)$ and by CO lines up to $J=22-21$; and 2) a compact hot component $(T \sim 1000 \mathrm{~K})$ traced by higher excitation $\mathrm{H}_{2} \mathrm{O}$ emission and higher- $J \mathrm{CO}$ transitions. Two gas components with different excitation conditions have also been proposed by Busquet et al. (2014) to account for the $\mathrm{H}_{2} \mathrm{O}$ and $\mathrm{CO}$ emission observed at the bright shock region B1 in the L1157 outflow. Finally, multiple-temperature components have been suggested to explain the spectrally unresolved $\mathrm{CO}$ ladder at the position of several Class 0 sources (e.g., Goicoechea et al. 2012; Herczeg et al. 2012; Dionatos et al. 2013; Karska et al. 2013; Manoj et al. 2013; Green et al. 2013). The nature of these two components and, notably, their spatial extent has not yet been clarified, however. To understand this problem better, maps of velocityresolved $\mathrm{H}_{2} \mathrm{O}$ lines that are sensitive to different excitation conditions are needed.

NGC $1333(d=235$ pc, Hirota et al. 2008) is a wellstudied star-forming region and contains many young stellar objects (YSOs) and outflows (e.g., Liseau et al. 1988; Bally et al. 1996; Knee \& Sandell 2000). Within the region, IRAS 4A and IRAS 4B are two low-mass protostars with a projected separation of about 30"; both have been identified as binary systems using mm interferometry (e.g., Lay et al. 1995; Looney et al. 2000; Choi 2005; Jørgensen et al. 2007). The companion to IRAS $4 \mathrm{~B}$ is detected at a separation of $11^{\prime \prime}$, whereas IRAS 4A is resolved into two components with a separation of only $2^{\prime \prime}$. IRAS $4 \mathrm{~A}$ is one of the youngest protobinary systems found so far, as inferred by its strong dust continuum emission with cold blackbody-like spectral energy distribution and its well-collimated outflow (e.g., Sandell et al. 1991; Blake et al. 1995), extending over arcminute scales. The IRAS 4A lowmass protostar has been the subject of extensive observations with ground-based submillimeter telescopes and interferometers (e.g., Blake et al. 1995; Di Francesco et al. 2001; Maret et al. 2005; Choi 2005; Jørgensen et al. 2007; Y1ld1z et al. 2012). As part of WISH, Kristensen et al. (2010, 2012) observed several $\mathrm{H}_{2} \mathrm{O}$ transitions towards the IRAS 4A source with HerschelHIFI, showing the complex line profiles with multiple components within the HIFI beam. In the line profiles, these authors identified a broad Gaussian component ( FWHM $\gtrsim 20 \mathrm{~km} \mathrm{~s}^{-1}$ ) that was also detected in the CO (10-9) emission (Y1ldiz et al. 2013) and is associated with the molecular outflow. In addition, they identified a so-called medium-broad component, offset with respect to the source velocity and with smaller line widths $\left(F W H M \sim 5-10 \mathrm{~km} \mathrm{~s}^{-1}\right)$, which they associated with currently shocked gas close to the protostar (Kristensen et al. 2013).

In this paper, we present new Herschel-PACS and HIFI observations of several key $\mathrm{H}_{2} \mathrm{O}$ lines that are sensitive to different excitation conditions, and HIFI CO (16-15) spectra at two shocked positions along the IRAS 4A outflow. The data are complemented by ground-based $\mathrm{CO}(3-2)$ and (6-5) maps by Y1ldiz et al. (2012). The goal is to study the spatial distribution of the water emission to spatially separate the multiple kinematic components that were previously detected towards the source within the HIFI beam. The observations and data reduction are described in Sect. 2. In Sect. 3 we present the observational results. The analysis and interpretation of the $\mathrm{H}_{2} \mathrm{O}$ excitation conditions and its physical origin are discussed in Sect. 4. Finally, in Sect. 5, we present the main conclusions.

\section{Observations}

\subsection{PACS observations}

PACS maps of $\mathrm{H}_{2} \mathrm{O}\left(2_{12}-1_{01}\right)$ at $1670 \mathrm{GHz}$ and $\mathrm{CO}(14-13)$ emission were used for the analysis. The maps are presented in Fig. 1, and a summary of the observations is given in Table 1. The observations are part of the OT1 program "Probing the physics and dynamics of the hidden warm gas in the youngest protostellar outflows" (OT1_bnisini_1). The PACS instrument is an Integral Field Unit (IFU), consisting of a $5 \times 5$ array of spatial pixels, each covering 9 .' $4 \times 99^{\prime \prime} 4$, for a total field of view of $47^{\prime \prime} \times$ $47^{\prime \prime}$. PACS was used in line-spectroscopy mode to obtain a spectral Nyquist-sampled raster map of IRAS 4A. The reference coordinates are at the position of IRAS $4 \mathrm{~A}, \alpha_{\mathrm{J} 2000}=03^{\mathrm{h}} 29^{\mathrm{m}} 10^{s} .50$, $\delta_{\mathrm{J} 2000}=+31^{\circ} 13^{\prime} 30^{\prime} \cdot 9$. The diffraction-limited FWHM beam size at $179 \mu \mathrm{m}$ is about $13^{\prime \prime}$. The data were reduced with HIPE ${ }^{1}$ (Herschel Interactive Processing Environment, Ott 2010) version 9.0. Within HIPE, they were flat-fielded and flux-calibrated by comparison with observations of Neptune. The calibration uncertainty is estimated to be around $20 \%$, based on the flux repeatability for multiple observations of the same target in different programs and on cross-calibration with HIFI and ISO. Finally, continuum subtraction was performed in IDL, and integrated line maps were obtained.

\subsection{HIFI observations}

We selected two active shock positions along the IRAS 4A outflow: R1 $\left(\alpha_{\mathrm{J} 2000}=03^{\mathrm{h}} 29^{\mathrm{m}} 10.82, \delta_{\mathrm{J} 2000}=+31^{\circ} 13^{\prime} 51^{\prime \prime} .9\right)$, at the origin of the jet from the driving source, and R2 $\left(\alpha_{\mathrm{J} 2000}=\right.$ $\left.03^{\mathrm{h}} 29^{\mathrm{m}} 14^{\mathrm{s}} 59, \delta_{\mathrm{J} 2000}=+31^{\circ} 14^{\prime} 45^{\prime \prime} .8\right)$, which is the head of the red lobe of the outflow. They appear as very bright peaks in the PACS $\mathrm{H}_{2} \mathrm{O}\left(2_{12}-1_{01}\right)$ and $\mathrm{CO}(14-13)$ maps shown in Fig. 1.

1 HIPE is a joint development by the Herschel Science Ground Segment Consortium, consisting of ESA, the NASA Herschel Science Center, and the HIFI, PACS and SPIRE consortia. 


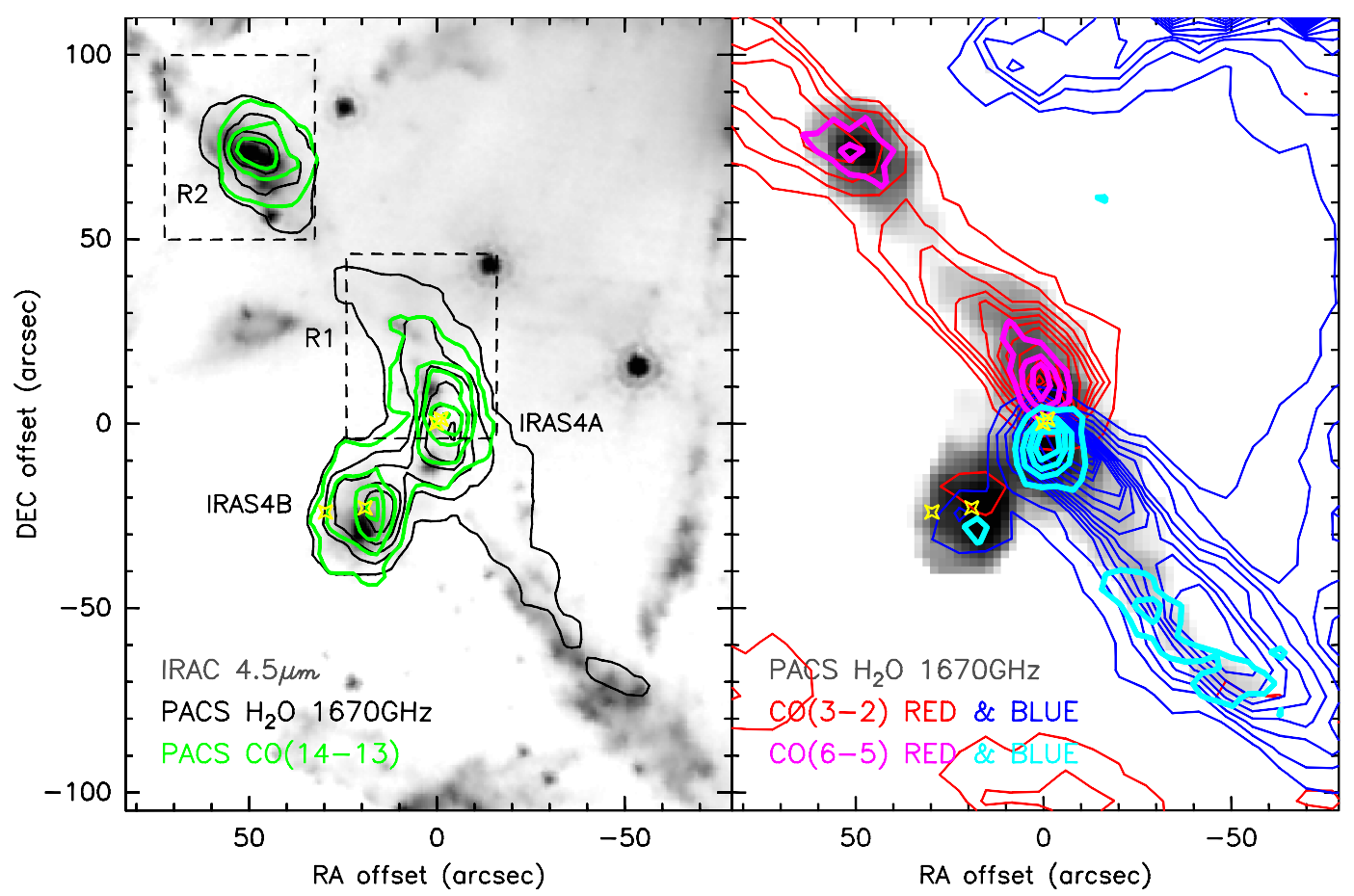

Fig. 1. PACS map of the $\mathrm{H}_{2} \mathrm{O}\left(2_{12}-1_{01}\right)$ emission at $1670 \mathrm{GHz}$ of the IRAS 4 region compared with the Spitzer-IRAC emission at $4.5 \mu$ m and the CO (14-13) emission in the left panel and with the JCMT CO (3-2) and APEX CO (6-5) emission (Yildız et al. 2012) in the right panel. The $\mathrm{CO}(3-2)$ and $\mathrm{CO}(6-5)$ maps are integrated in the velocity ranges between $-20 \mathrm{~km} \mathrm{~s}^{-1}$ and $3 \mathrm{~km} \mathrm{~s}^{-1}$ for the blue-shifted emission and $12 \mathrm{~km} \mathrm{~s}{ }^{-1}$ and $50 \mathrm{~km} \mathrm{~s}^{-1}$ for the red-shifted emission. The contour levels start at the $5 \sigma$ level and increase in steps of $10 \sigma$ for the $\mathrm{PACS} \mathrm{H}_{2} \mathrm{O}$ and $\mathrm{CO}(14-13)$ maps, from the $5 \sigma$ level emission in steps of $5 \sigma$ for the $\mathrm{CO}(3-2)$ and from the $5 \sigma$ level emission in steps of $3 \sigma$ for the $\mathrm{CO}(6-5)$. Offsets are with respect to the central source IRAS 4A, at coordinates $\alpha_{\mathrm{J} 2000}=03^{\mathrm{h}} 29^{\mathrm{m}} 10.50, \delta_{\mathrm{J} 2000}=+31^{\circ} 13^{\prime} 30^{\prime} 9$. The positions of the IRAS 4A and IRAS 4B binary sources are marked with yellow symbols (Looney et al. 2000). The regions mapped with HIFI in the water lines around the selected shock positions (R1 and R2) are indicated.

Table 1. Parameters of the lines mapped with PACS.

\begin{tabular}{lcccc}
\hline \hline Line & $\begin{array}{c}\text { Frequency } \\
(\mathrm{GHz})\end{array}$ & $\begin{array}{c}\text { Wavelength } \\
(\mu \mathrm{m})\end{array}$ & $\begin{array}{c}E_{\mathrm{u}} / k_{\mathrm{B}} \\
(\mathrm{K})\end{array}$ & $\begin{array}{c}H P B W \\
(\operatorname{arcsec})\end{array}$ \\
\hline $\mathrm{o}-\mathrm{H}_{2} \mathrm{O}\left(2_{12}-1_{01}\right)$ & 1669.90 & 179.5 & 114.4 & 13 \\
$\mathrm{CO}(14-13)$ & 1611.79 & 186.0 & 580.5 & 13 \\
\hline
\end{tabular}

Notes. The observation IDs of both lines are 1342225852, 1342225853, and 1342225854 .

Single-pointing observations of the o- $\mathrm{H}_{2} \mathrm{O}\left(1_{10}-1_{01}\right)$ line at $557 \mathrm{GHz}$ and the $\mathrm{CO}(16-15)$ transition at the two selected positions were conducted with Herschel-HIFI in dual beamswitch and fast-chop mode. In addition, an area of size equal to the HIFI beam width at $557 \mathrm{GHz}\left(38^{\prime \prime}\right)$ was mapped in onthe-fly mode in three other $\mathrm{H}_{2} \mathrm{O}$ lines, spanning excitation energies $E_{\mathrm{u}}$ from $50 \mathrm{~K}$ to $250 \mathrm{~K}$ (see Table 2). The observations were carried out between July 2012 and August 2012 as part of the OT2 program "Solving the puzzle of water excitation in shocks" (OT2_gsantang_1). Contextually, the spectral set-up allowed us to observe transitions from other molecules: $\mathrm{N}_{2} \mathrm{H}^{+}(6-5)$, SO $\left(13_{14}-12_{13}\right), \mathrm{CH}_{3} \mathrm{OH}\left(3_{-2}-2_{-1}\right), \mathrm{NH}_{3}\left(1_{0}-0_{0}\right)$, and ${ }^{13} \mathrm{CO}$ (10-9). A summary of the performed observations is given in Tables 2 and A.1.

The data were processed with the ESA-supported package HIPE version 11 for calibration. The calibration uncertainty is taken to be $20 \%$. Further reduction of all the spectra, including baseline subtraction, and the analysis of the data were performed using the GILDAS ${ }^{2}$ software. H- and V-polarizations were coadded after inspection to increase sensitivity, since no significant differences were found between the two data sets. The calibrated $T_{\mathrm{A}}^{*}$ scale from the telescope was converted into the $T_{\mathrm{mb}}$ scale using the main-beam efficiency factors provided by Roelfsema et al. $(2012)^{3}$ and reported in Table 2 . At the velocity resolution of $1 \mathrm{~km} \mathrm{~s}^{-1}$, the rms noise ranges between $10 \mathrm{mK}$ and $20 \mathrm{mK}$ ( $T_{\mathrm{mb}}$ scale $)$.

\section{Results}

Figure 1 shows the PACS maps of $\mathrm{H}_{2} \mathrm{O}\left(2_{12}-1_{01}\right)$ at $1670 \mathrm{GHz}$ and CO (14-13), in comparison with the Spitzer-IRAC emission at $4.5 \mu \mathrm{m}$ and the ground-based $\mathrm{CO}(3-2)$ and $\mathrm{CO}(6-5)$ (Yildiz et al. 2012) towards the IRAS 4 region. The figure highlights the spatial correlation between $\mathrm{H}_{2} \mathrm{O}$, high- $J \mathrm{CO}$, and Spitzer $4.5 \mu \mathrm{m}$ emissions, in agreement with previous studies of outflows from low-mass protostars (e.g., Nisini et al. 2010; Santangelo et al. 2013; Tafalla et al. 2013). In particular, PACS CO (14-13) and APEX CO (6-5) emissions are spatially associated with the PACS $\mathrm{H}_{2} \mathrm{O}$ emission, whereas the low- $J \mathrm{CO}(3-2)$ emission is more extended and offset. A sharp change of propagation direction of $\sim 30^{\circ}$ in the north-eastern outflow red lobe, occurring close to the $\mathrm{R} 1$ shock position, is visible both in $\mathrm{H}_{2} \mathrm{O}$ and $\mathrm{CO}$ emission. This directional variability was previously shown by both single-dish and interferometric observations, and several mechanisms were proposed to explain it, including magnetic

\footnotetext{
2 http://www.iram.fr/IRAMFR/GILDAS/

3 See also http://herschel.esac.esa.int/twiki/bin/view/ Public/ HifiCalibrationWeb?template=viewprint.
} 

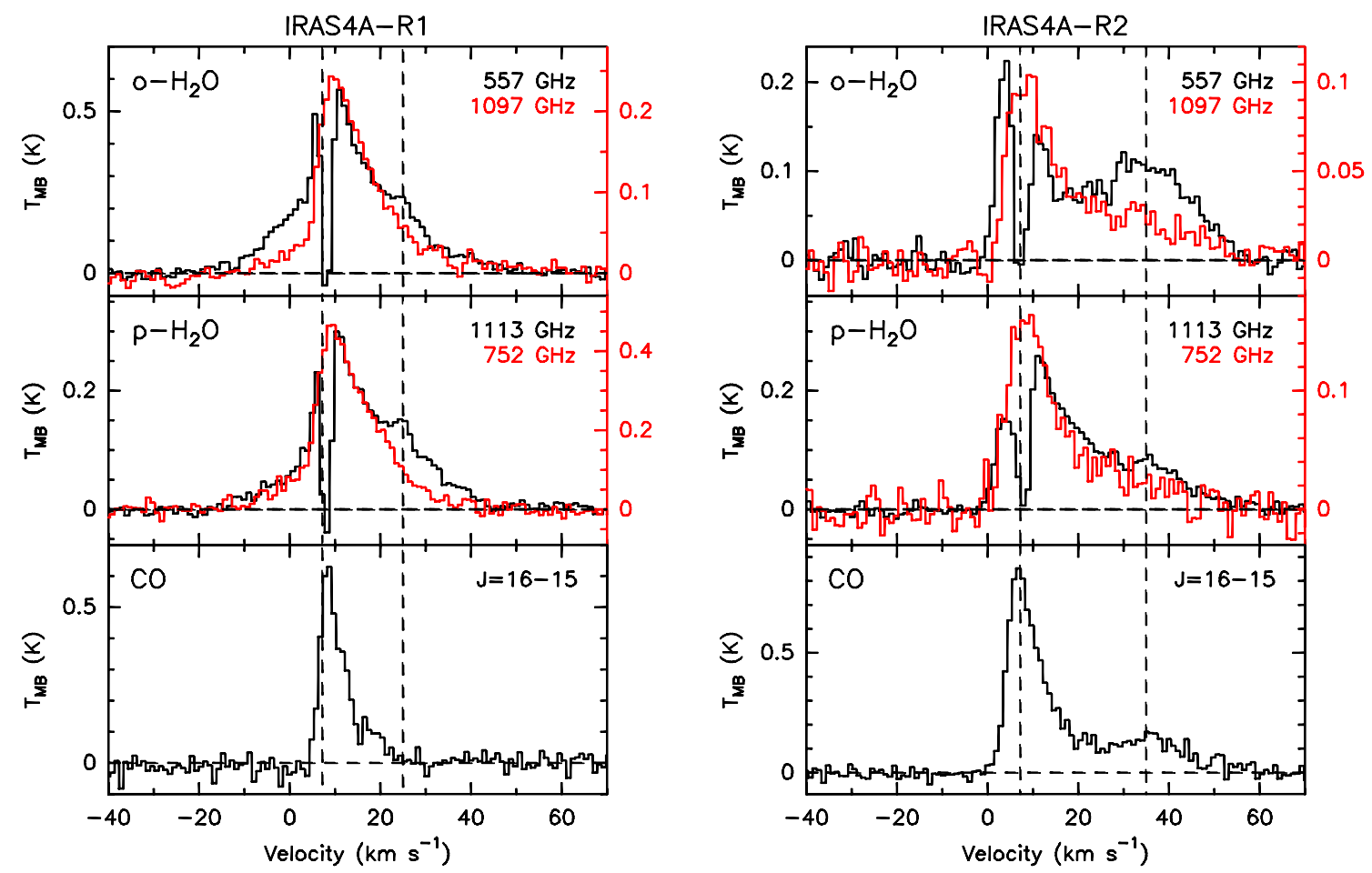

Fig. 2. HIFI spectra of the $\mathrm{H}_{2} \mathrm{O}$ and $\mathrm{CO}$ transitions observed at the R1 (left) and R2 (right) shock positions along IRAS 4A. The spectra are all convolved to the same angular resolution of the $\mathrm{H}_{2} \mathrm{O}\left(1_{10}-1_{01}\right)$ line at $557 \mathrm{GHz}\left(\sim 38^{\prime \prime}\right)$, with the exception of the $\mathrm{CO}(16-15)$ line, which is a single pointing HIFI observation at $\sim 12^{\prime \prime}$ resolution. The spectra shown in red have intensities provided in the right-hand axes. The vertical dashed line marks the systemic velocity $\left(v_{\mathrm{LSR}}=+7.2 \mathrm{~km} \mathrm{~s}^{-1}\right)$ and the $\mathrm{H}_{2} \mathrm{O}$ secondary emission peaks at +25 and $+35 \mathrm{~km} \mathrm{~s}^{-1}$ for R1 and R2 .

Table 2. Parameters of the lines observed with HIFI.

\begin{tabular}{lcccccrrr}
\hline \hline Line & Observation ID & Band & Mode $^{a}$ & Sideband & $\eta_{\mathrm{MB}}$ & $\begin{array}{r}\text { Frequency } \\
(\mathrm{MHz})\end{array}$ & $\begin{array}{r}E_{\mathrm{u}} / k_{\mathrm{B}} \\
(\mathrm{K})\end{array}$ & $\begin{array}{r}H P B W \\
(\operatorname{arcsec})\end{array}$ \\
\hline $\mathrm{o}-\mathrm{H}_{2} \mathrm{O}\left(1_{10}-1_{01}\right)$ & 1342248895,1342248897 & 1 & $\mathrm{SP}$ & $\mathrm{LSB}$ & 0.75 & 556936.07 & 61 & 38 \\
$\mathrm{p}-\mathrm{H}_{2} \mathrm{O}\left(2_{11}-2_{02}\right)$ & 1342249432,1342249433 & 2 & $\mathrm{M}$ & $\mathrm{USB}$ & 0.75 & 752033.23 & 137 & 28 \\
$\mathrm{o}-\mathrm{H}_{2} \mathrm{O}\left(3_{12}-3_{03}\right)$ & 1342249853,1342249854 & 4 & $\mathrm{M}$ & $\mathrm{USB}$ & 0.74 & 1097365.05 & 249 & 19 \\
$\mathrm{p}-\mathrm{H}_{2} \mathrm{O}\left(1_{11}-0_{00}\right)$ & 1342249021,1342250209 & 4 & $\mathrm{M}$ & $\mathrm{USB}$ & 0.74 & 1113343.06 & 53 & 19 \\
$\mathrm{CO}(16-15)$ & 1342249639,1342249640 & 7 & $\mathrm{SP}$ & $\mathrm{USB}$ & 0.70 & 1841345.51 & 752 & 12 \\
\hline
\end{tabular}

Notes. ${ }^{(a)} \mathrm{SP}=$ Single Pointing mode; $\mathrm{M}=$ Mapping mode.

deflection, a precessing jet, and collisions with a dense core in the ambient cloud (e.g., Blake et al. 1995; Girart et al. 1999; Choi 2001, 2005; Baek et al. 2009; Choi et al. 2011; Y1ld1z et al. 2012). Strong $\mathrm{H}_{2} \mathrm{O}$ emission peaks are found at the location of active shocked regions and at the position of IRAS 4A and its neighbour IRAS 4B (see also Nisini et al. 2010, 2013). In particular, the R1 and R2 shock positions appear as bright peaks in the $\mathrm{H}_{2} \mathrm{O}$ and high- $J$ CO emission, as revealed by PACS and ground-based observations.

An overview of the HIFI observations is given in Fig. 2 and Fig. A.1, where $\mathrm{H}_{2} \mathrm{O}$ and $\mathrm{CO}$ spectra observed at the two selected shock positions and the spectra of additional lines detected with HIFI are shown. All $\mathrm{H}_{2} \mathrm{O}$ spectra observed in mapping mode are convolved to the same angular resolution of $38^{\prime \prime}$ for comparison with the observations of the ground-state $\mathrm{o}-\mathrm{H}_{2} \mathrm{O}$ transition at $557 \mathrm{GHz}$. Several kinematic components can be distinguished in the observed $\mathrm{H}_{2} \mathrm{O}$ line profiles at both the $\mathrm{R} 1$ and $\mathrm{R} 2$ positions. First, an absorption dip around the systemic velocity of IRAS $4 \mathrm{~A}\left(v_{\mathrm{LSR}}=+7.2 \mathrm{~km} \mathrm{~s}^{-1}\right.$, Kristensen et al. 2012) is detected in the ground-state transitions of o- and $\mathrm{p}-\mathrm{H}_{2} \mathrm{O}$ (at
$557 \mathrm{GHz}$ and $1113 \mathrm{GHz}$ ), associated with cold gas in the outer envelope. Second, a triangularly shaped outflow wing is present up to about $50 \mathrm{~km} \mathrm{~s}^{-1}$ at R1 and about $60 \mathrm{~km} \mathrm{~s}^{-1}$ at R2. Third, an excess of emission at high velocity is observed in the groundstate transitions of o- and $\mathrm{p}-\mathrm{H}_{2} \mathrm{O}$. This secondary high-velocity (HV) emission peak appears at a velocity of about $+25 \mathrm{~km} \mathrm{~s}^{-1}$ and $+35 \mathrm{~km} \mathrm{~s}^{-1}$ in R1 and R2. Similar variations of water line profiles with excitation were observed at the bow-shock positions along the red lobes of the L1448 (R4) and L1157 (R) outflows by Santangelo et al. (2012) and Vasta et al. (2012).

Figure 3 presents the $\mathrm{H}_{2} \mathrm{O} 557 \mathrm{GHz} / 1097 \mathrm{GHz}$ line ratio as a function of velocity for R1 and R2. Water transitions with different upper level energies were chosen and convolved to the same angular resolution of $38^{\prime \prime}$, and the ratios are plotted only for velocities where both transitions have $S / N>3$. Neglecting the velocity range $4-10 \mathrm{~km} \mathrm{~s}^{-1}$, where the absorption dip in the $557 \mathrm{GHz} \mathrm{H}{ }_{2} \mathrm{O}$ contaminates the analysis, the ratio between the o$\mathrm{H}_{2} \mathrm{O}\left(1_{10}-1_{01}\right)$ and $\mathrm{o}-\mathrm{H}_{2} \mathrm{O}\left(3_{12}-3_{03}\right)$ lines increases significantly with velocity, reflecting the fact that the secondary $\mathrm{HV}$ peak at both R1 and R2 appears in the lower excitation energy transitions 

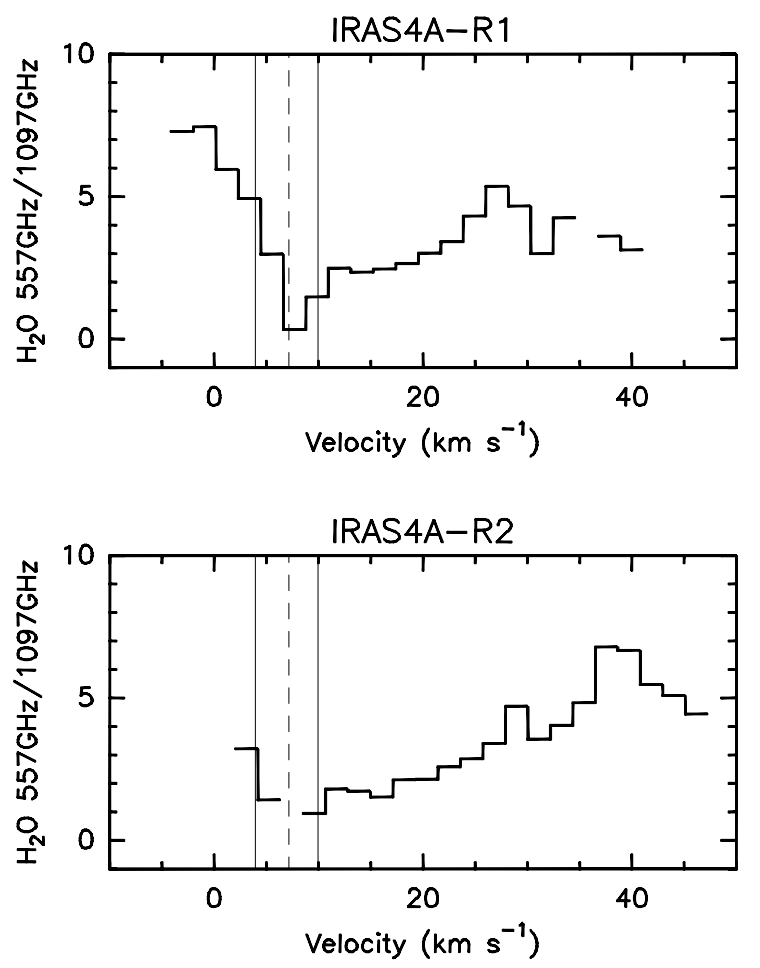

Fig. 3. Ratio between the o- $\mathrm{H}_{2} \mathrm{O}\left(1_{10}-1_{01}\right)$ (at $557 \mathrm{GHz}$ ) and o- $\mathrm{H}_{2} \mathrm{O}$ $\left(3_{12}-3_{03}\right)$ (at $1097 \mathrm{GHz}$ ) lines as a function of velocity at the two observed shock positions R1 (upper panel) and R2 (lower panel). The $1097 \mathrm{GHz}$ water spectra are convolved to the same angular resolution as the $557 \mathrm{GHz}$ spectra, i.e., $38^{\prime \prime}$. The line ratios are plotted only where the $\mathrm{S} / \mathrm{N}$ ratio is higher than three for the two lines, at a spectral resolution of $2 \mathrm{~km} \mathrm{~s}^{-1}$. The vertical dashed line indicates the source velocity, whereas the solid vertical lines mark the velocity range of the absorption dip $\left(4-10 \mathrm{~km} \mathrm{~s}^{-1}\right)$.

(see Fig. 2). Finally, we note that the increasing trend is stronger at R2, the shock position farthest from the central driving source. We point out that these findings for the shock positions are in contrast to observations of $\mathrm{H}_{2} \mathrm{O}$ emission lines at the central protostellar positions, which show constant line ratios (Mottram et al., in prep.).

Recent studies have shown that high- $J$ CO emission is associated with $\mathrm{H}_{2} \mathrm{O}$ emission, corresponding to a warm ( $\gtrsim 300 \mathrm{~K}$ ) and dense $\left(n_{\mathrm{H}_{2}} \gtrsim 10^{6} \mathrm{~cm}^{-3}\right)$ gas component (e.g., Karska et al. 2013; Santangelo et al. 2013). This finding is consistent with our CO (16-15) observations at R2, showing a similar line profile as the ground-state water transitions, that is, strong, broad emission around the systemic velocity of the source and an $\mathrm{HV}$ emission peak. We note that the detection of a secondary $\mathrm{HV}$ emission component at $\mathrm{R} 2$ in the $\mathrm{H}_{2} \mathrm{O} 557 \mathrm{GHz}$ and in the $\mathrm{CO}(16-15)$ lines, with angular resolutions of about $38^{\prime \prime}$ and $12^{\prime \prime}$, suggests that this emission is associated with a compact gas component centred on the shock. Therefore, the non-detection of this HV emission peak in the higher-excitation, smaller-beam size $\mathrm{H}_{2} \mathrm{O}$ transitions is probably due to an excitation effect, with the HV component being less excited than the low-velocity (LV) component, as previously found in the L1448 and L1157 bow shock positions (e.g., Vasta et al. 2012; Santangelo et al. 2012).

On the other hand, at the R1 position no clear secondary peak around $+25 \mathrm{~km} \mathrm{~s}^{-1}$ is detected in CO (16-15). This difference possibly arises because the HV peak is spatially shifted towards IRAS 4 A by $\sim 10^{\prime \prime}$ with respect to R1, while the CO (16-15) was observed with a beam size of $12^{\prime \prime}$ (see also Sect. 3.1 and Figs. 7 and 8).

\subsection{Water spatial distribution}

Figure 4 shows the HIFI spectra at $1113 \mathrm{GHz}$ observed around the R1 and R2 shock positions. The figure highlights the variation of the water line profiles around the shocked regions.

From examining the $\mathrm{R} 1$ position, we note that the $\mathrm{H}_{2} \mathrm{O}$ line profiles close to IRAS 4A resemble those along the outflow, which testifies that the outflow dominates the water profiles. This similarity is even clearer when we compare the $\mathrm{H}_{2} \mathrm{O}$ profiles centred at R1 and at IRAS 4A (Fig. 5). The $\mathrm{p}-\mathrm{H}_{2} \mathrm{O} 1113 \mathrm{GHz}$ spectra show the same profile in the red-shifted emission, although the on-source position is brighter than $\mathrm{R} 1$. On the other hand, the $\mathrm{H}_{2} \mathrm{O}$ profiles of the higher excitation energy $\mathrm{H}_{2} \mathrm{O} 1097 \mathrm{GHz}$ line appear to be different, with the on-source position being fainter than R1. This difference suggests that at R1 the excitation conditions of the $\mathrm{H}_{2} \mathrm{O}$ emitting gas are different from the conditions at the position of the central source. In addition, in the on-source spectra, significant blue-shifted emission is detected that is not seen at the R1 position. The blue wing presents an excess of emission at $\sim 0 \mathrm{~km} \mathrm{~s}^{-1}\left(-7 \mathrm{~km} \mathrm{~s}^{-1}\right.$ with respect to the source velocity), which is quite symmetric in the $1113 \mathrm{GHz}$ line with respect to the red-shifted secondary peak. Kristensen et al. (2013) interpreted this blue-shifted $\mathrm{H}_{2} \mathrm{O}$ component as originating from a compact dissociative shock close to the protostar. We remark that our profiles present some differences with those presented in Kristensen et al. (2013); in the latter, for example, the secondary red-shifted peak is not as bright and the relative intensity of the low- and high-velocity blue-shifted components is significantly different. These differences are probably due to the different observation pointings. However, Kristensen et al. (2013) suggested that time variability might also change the $\mathrm{H}_{2} \mathrm{O}$ line profiles.

The R2 position, which is a pure shock position far from the driving source of the outflow, is a very interesting laboratory to study the water distribution around shocks. Our HIFI observations clearly show that the different water kinematic components mentioned above (see Sect. 3 and Fig. 2) are not uniformly distributed across the mapped region (Fig. 4). In particular, the $\mathrm{HV}$ peak is observed only at the bright shock peak, but is not detected in water line profiles offset from the shock position, which show a triangular wing shape. These two types of line profiles probably indicate two different gas components in the water emission: a compact gas component, located at the shock peak and associated with the HV peak, and a more diffuse component. The difference is shown in the left panel of Fig. 6, where line emission from the $\mathrm{R} 2$ position and a position $\left(10^{\prime \prime},-10^{\prime \prime}\right)$ offset from the $\mathrm{R} 2$ shock are chosen as representative. An emission residual between the two spectra is also displayed. The spectrum associated with the compact component shows an excess of emission at the systemic velocity and at the HV peak with respect to the extended component. The two detected components are therefore not kinematically distinct, since the velocity range of the emission is the same in both cases. Thus, it would not have been possible to distinguish them within the $38^{\prime \prime}$ beam size of single-pointing observations.

In Fig. 7, the maps of the $\mathrm{H}_{2} \mathrm{O} 1113 \mathrm{GHz}$ emission, integrated in the three velocity ranges reported in the caption and corresponding to blue-shifted emission, LV red-wing emission, and HV emission peak, are presented for the R1 (upper panel) and the R2 (lower panel) positions, in comparison with the $\mathrm{H}_{2} \mathrm{O}$ $1670 \mathrm{GHz}$ PACS map. Blue-shifted emission is only detected close to the central source IRAS 4A. At R1, the HV-peak emission appears to have a compact distribution, spatially associated with IRAS 4A, whereas the LV-wing emission is elongated in the outflow red-lobe direction. In contrast, no significant difference 

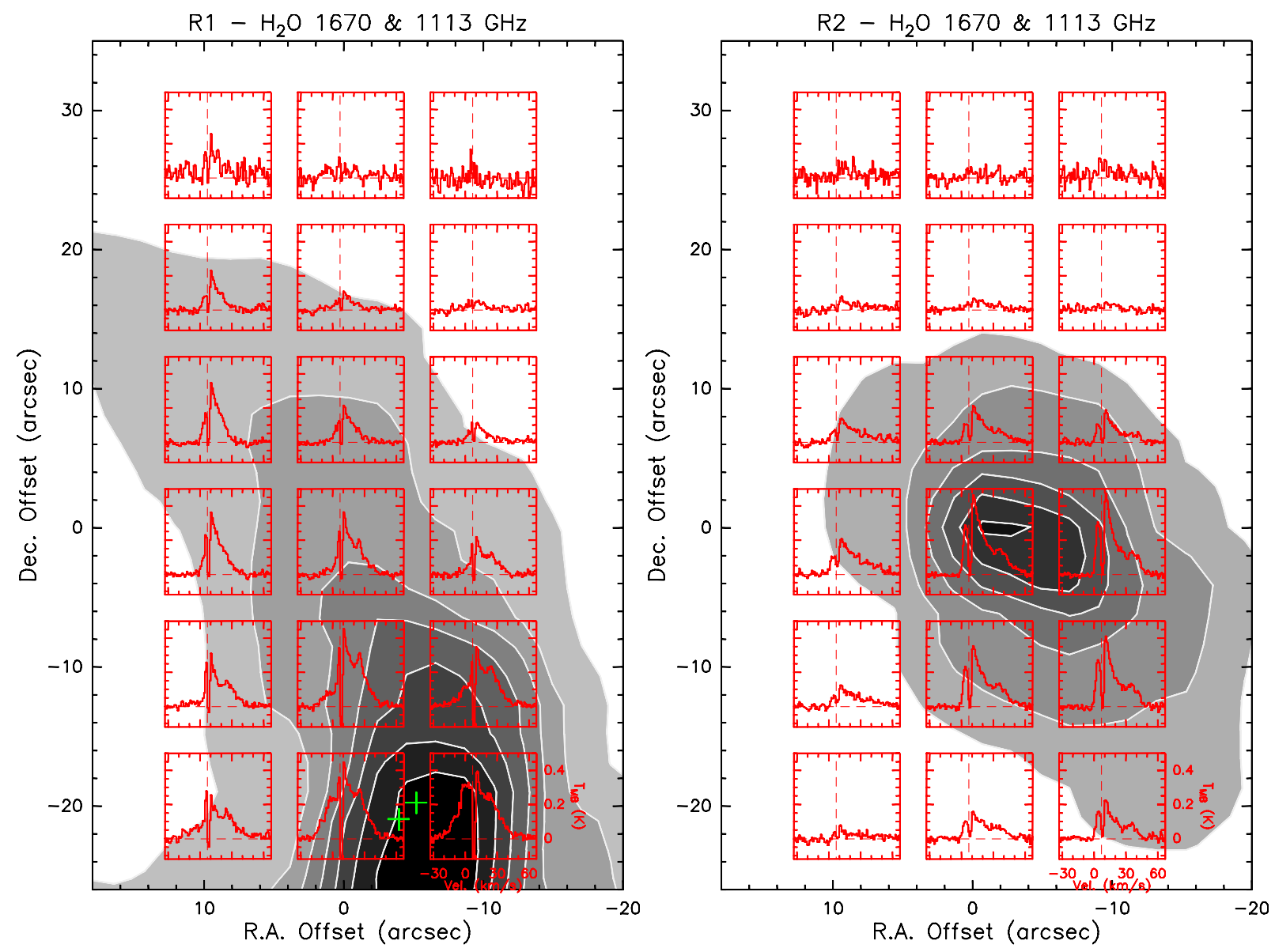

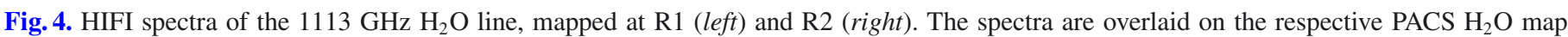
at $1670 \mathrm{GHz}$ (grey scale and white contours). Offsets are with respect to the R1 and R2 shock positions. The IRAS 4A binary source position is marked with green crosses.

between the LV-wing and the HV-peak emission can be observed at R2 at this angular resolution (19", corresponding to about $4500 \mathrm{AU})$ with both distributions appearing unresolved. This difference is discussed in more detail in Sect. 4.

Similar conclusions can be drawn at R1 from Fig. 8, where the $\mathrm{CO}(3-2)$ emission is integrated in the same velocity ranges as adopted for the $\mathrm{H}_{2} \mathrm{O}$ maps of Fig. 7. The HV-peak emission appears to be more compact than the LV-wing emission and shifted with respect to R1 towards IRAS 4A. We note that a second north-eastern emission peak can be identified in the HV-peak emission, which is not associated with bright PACS $\mathrm{H}_{2} \mathrm{O} 179 \mu \mathrm{m}$ emission. A different situation with respect to the $\mathrm{H}_{2} \mathrm{O} 1113 \mathrm{GHz}$ emission is observed at R2 in the CO (3-2) emission, however. Here, the HV-peak emission shows a compact distribution that is not resolved on an angular scale of $14^{\prime \prime}$ and spatially associated with the $\mathrm{H}_{2} \mathrm{O}$ emission at $179 \mu \mathrm{m}$. In contrast, the LV-wing emission is associated with a diffuse gas component, following the outflow direction, and is spatially more extended than the $\mathrm{H}_{2} \mathrm{O}$ emission. The $\mathrm{CO}$ (3-2) observations thus seem to support the scenario of two distinct gas components in the $\mathrm{R} 2$ shock position.

Finally, a comparison between the CO (3-2) spectra observed at R2 and at a position $\left(10^{\prime \prime},-10^{\prime \prime}\right)$ offset from the shock is presented in the right panel of Fig. 6. Although the $\mathrm{CO}(3-2)$ spectra show different line profiles in the LV emission with respect to $\mathrm{H}_{2} \mathrm{O}$, once more, they indicate that the $\mathrm{HV}$ peak is

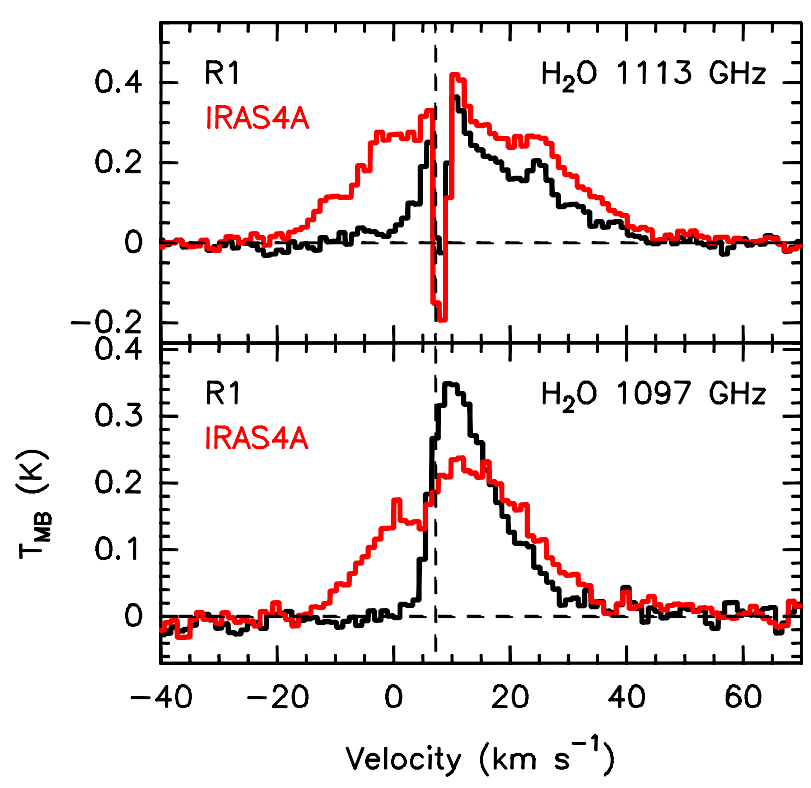

Fig. 5. Comparison between the $\mathrm{H}_{2} \mathrm{O}\left(1_{11}-0_{00}\right)(1113 \mathrm{GHz})$ and $\left(3_{12}-3_{03}\right)(1097 \mathrm{GHz})$ line profiles at R1 and on the IRAS 4A source. The vertical dashed line indicates the source velocity.

associated with a compact gas component not detected in the more diffuse LV gas. 
G. Santangelo et al.: Water distribution in shocked regions of NGC 1333-IRAS 4A
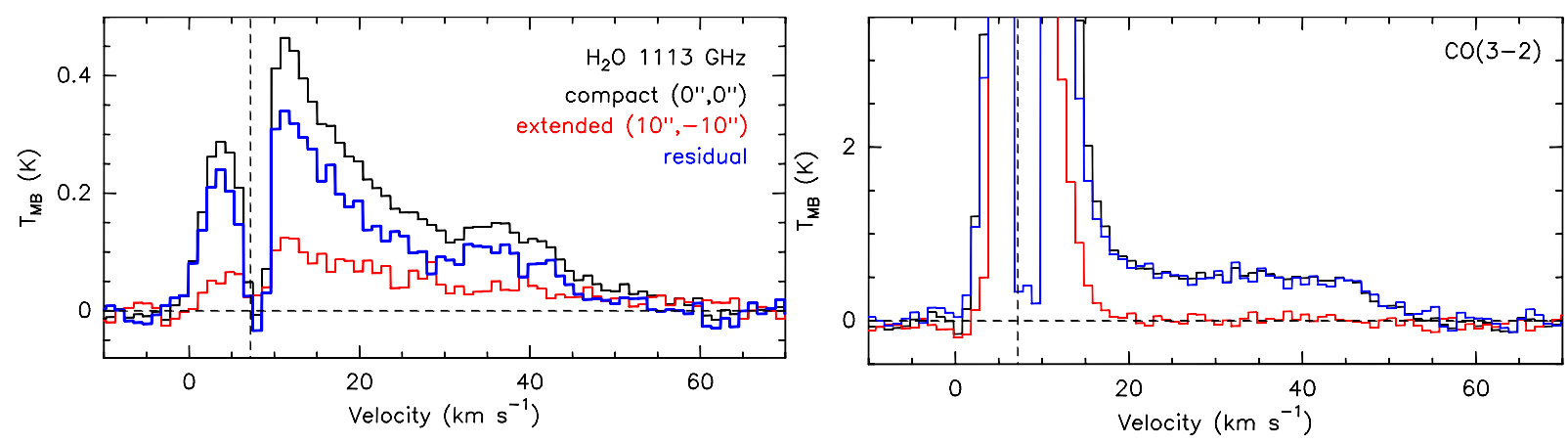

Fig. 6. Left: comparison between the $\mathrm{H}_{2} \mathrm{O} 1_{11}-0_{00}$ emission at $1113 \mathrm{GHz}$ (19" beam size) at the $\mathrm{R} 2$ shock position (black), corresponding to the compact gas component, and that at the offset position $\left(10^{\prime \prime},-10^{\prime \prime}\right)$ with respect to R2 (red), corresponding to the extended component. The residual spectrum, given by the difference between the two displayed spectra, is shown in blue. Offsets are with respect to R2. The vertical dashed line indicates the source velocity. Right: same as the left panel for the $\mathrm{CO}(3-2)$ emission.
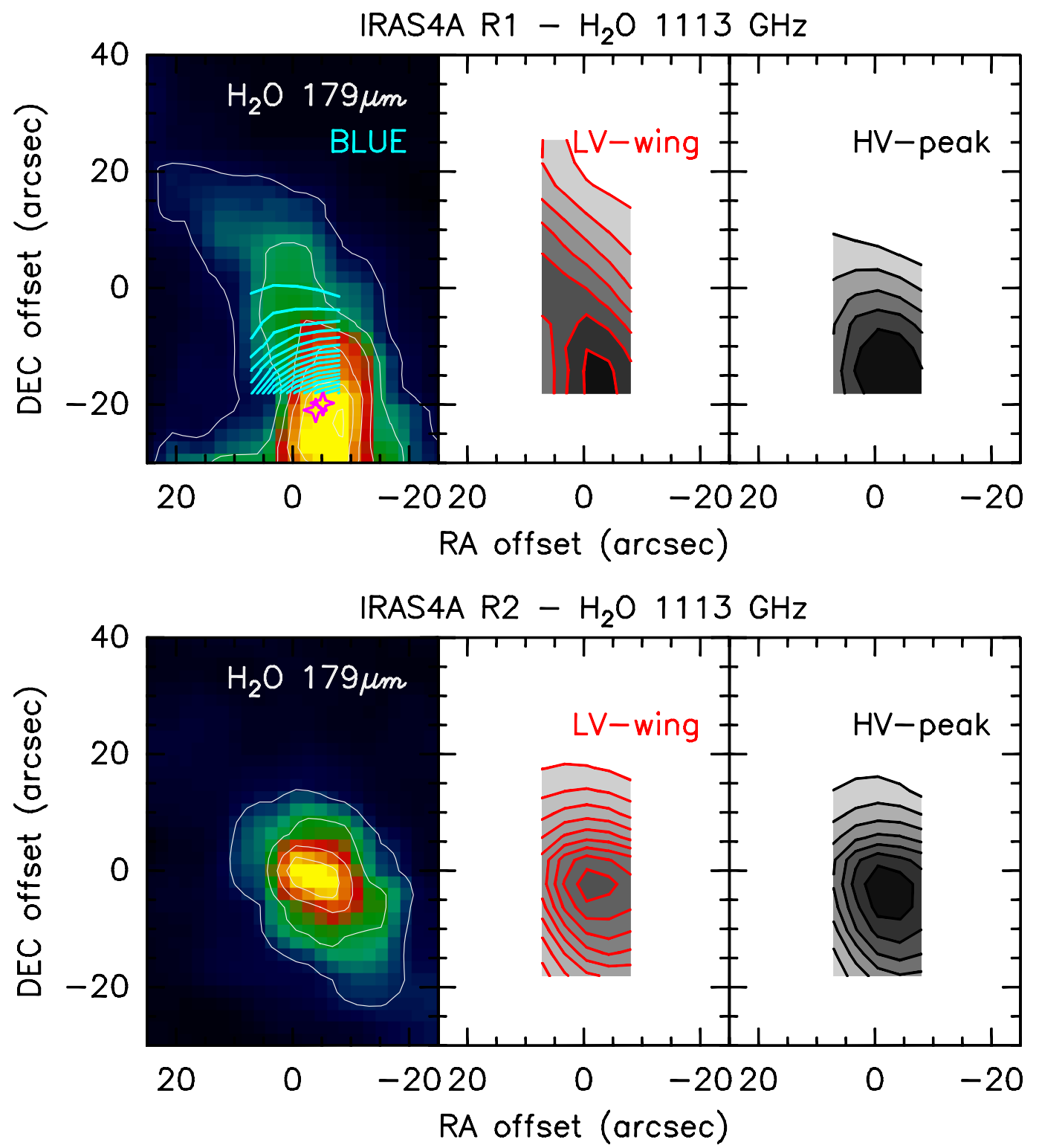

Fig. 7. Velocity-integrated maps of the HIFI $\mathrm{H}_{2} \mathrm{O} 1113 \mathrm{GHz}$ emission at the R1 (upper panel) and R2 (lower panel) positions compared with the PACS $\mathrm{H}_{2} \mathrm{O} 179 \mu \mathrm{m}$ map. At R1, the $\mathrm{H}_{2} \mathrm{O} 1113 \mathrm{GHz}$ emission is integrated in three velocity ranges: the blue-shifted emission (between $-20 \mathrm{~km} \mathrm{~s}^{-1}$ and $3 \mathrm{~km} \mathrm{~s}^{-1}$ ), the low-velocity (LV) wing emission (between $11 \mathrm{~km} \mathrm{~s}^{-1}$ and $20 \mathrm{~km} \mathrm{~s}^{-1}$ ), and the high-velocity (HV) emission peak (between $20 \mathrm{~km} \mathrm{~s}^{-1}$ and $30 \mathrm{~km} \mathrm{~s}^{-1}$ ). At R2, two velocity ranges are considered: the LV wing emission (between $11 \mathrm{~km} \mathrm{~s}^{-1}$ and $30 \mathrm{~km} \mathrm{~s}^{-1}$ ) and the HV emission peak (between $30 \mathrm{~km} \mathrm{~s}^{-1}$ and $45 \mathrm{~km} \mathrm{~s}^{-1}$ ). The contour levels start from the $5 \sigma$ level and increase in steps of $10 \sigma$ for the PACS $179 \mu \mathrm{m}$ emission and in steps of $3 \sigma$ for the HIFI $1113 \mathrm{GHz}$ emission. Offsets are with respect to the R1 and R2 shock positions. The magenta symbols represent the position of the IRAS 4A binary source. 

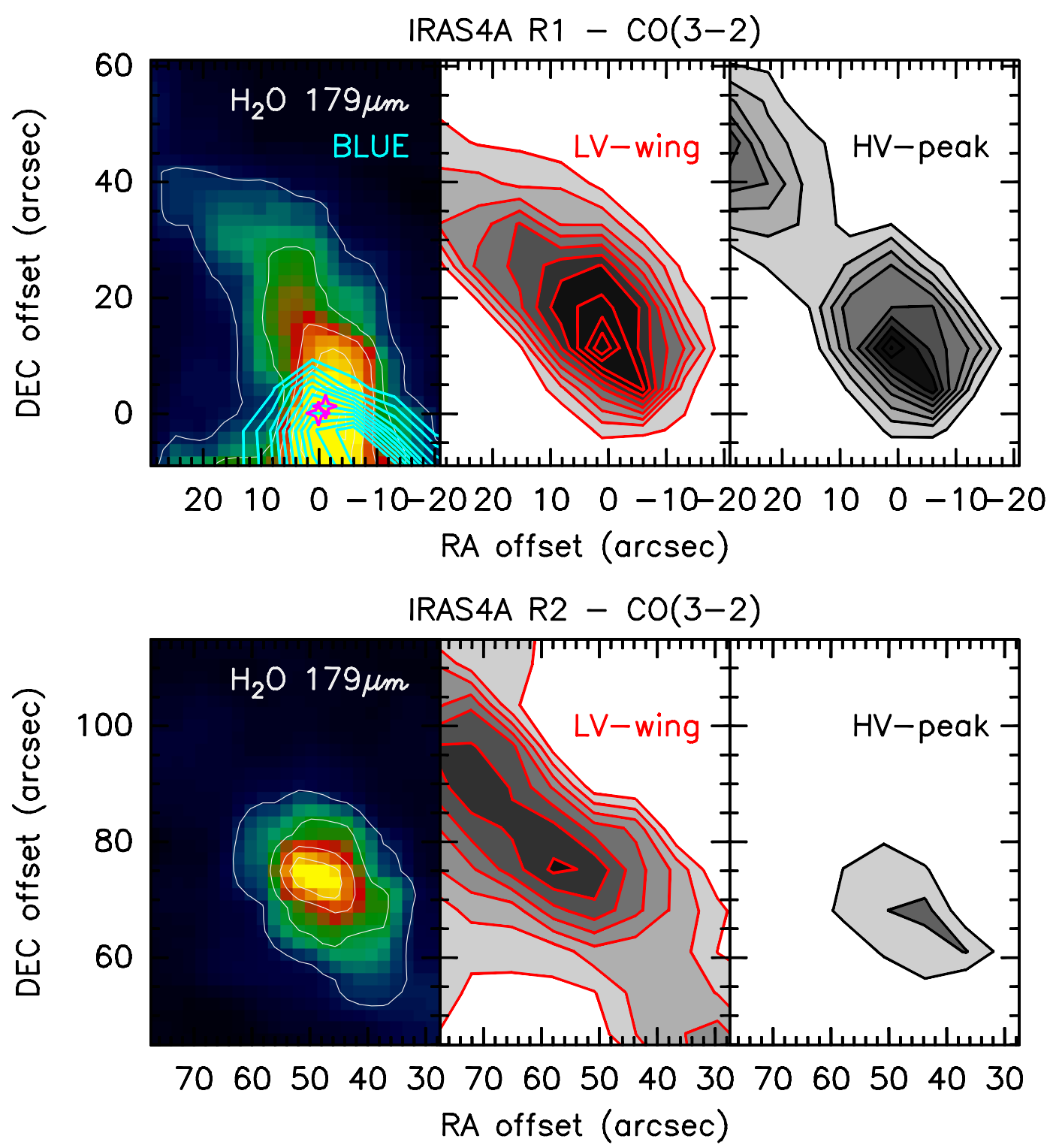

Fig. 8. Same as Fig. 7 for the CO (3-2) emission. Offsets here are with respect to the central source IRAS 4A.

We note that, because of the low sensitivity in the line wings, the APEX CO (6-5) data do not detect HV line emission and thus cannot be used to analyse the two spatial components observed in the $\mathrm{H}_{2} \mathrm{O}$ and $\mathrm{CO}(3-2)$ lines.

\section{Two gas components in shocked $\mathrm{H}_{2} \mathrm{O}$ emission}

To investigate the presence of two distinct gas components at $\mathrm{R} 2$, we compared their associated $\mathrm{H}_{2} \mathrm{O}$ line ratios with respect to the higher excitation energy $\mathrm{H}_{2} \mathrm{O} 1097 \mathrm{GHz}$ line (Fig. 9). We assumed that the compact gas component dominates the $\mathrm{H}_{2} \mathrm{O}$ emission at the $\mathrm{R} 2$ shock position while the extended component dominates the $\mathrm{H}_{2} \mathrm{O}$ emission at the position $\left(10^{\prime \prime},-10^{\prime \prime}\right)$ offset from R2, as supported by the difference in the observed line profiles (see Figs. 4 and 6). The line intensities are integrated only in the line wings, between $10 \mathrm{~km} \mathrm{~s}^{-1}$ and $60 \mathrm{~km} \mathrm{~s}^{-1}$, because of the absorption dip at the source systemic velocity. The two distinct gas components with different excitation conditions at R2 are confirmed by the significantly different associated water line ratios. In particular, the three measured line ratios are higher in the compact component, which is consistent with this component being less excited than the extended one and detected only in the lower excitation water lines.

To characterize the two gas components at R2 in terms of excitation conditions, we ran the radex non-LTE molecular LVG radiative transfer code (van der Tak et al. 2007) in plane-parallel geometry, with collisional rate coefficients from Dubernet et al. (2006, 2009) and Daniel et al. (2010, 2011) and molecular data from the Leiden Atomic and Molecular Database (LAMDA ${ }^{4}$, Schöier et al. 2005). A grid of models with density ranging between $10^{4} \mathrm{~cm}^{-3}$ and $10^{8} \mathrm{~cm}^{-3}$ and temperatures ranging between $100 \mathrm{~K}$ and $1600 \mathrm{~K}$ was built. Two values of o- $\mathrm{H}_{2} \mathrm{O}$ column density were considered, corresponding to optically thin and moderately thick water emission. We adopted a typical line width of $20 \mathrm{~km} \mathrm{~s}^{-1}$ for both components from the HIFI spectra and an $\mathrm{H}_{2} \mathrm{O}$ ortho-to-para ratio equal to 3 (Emprechtinger et al. 2013), corresponding to the high-temperature equilibrium value. The ratio between the $\mathrm{p}-\mathrm{H}_{2} \mathrm{O} 1113 \mathrm{GHz}$ and the $\mathrm{o}-\mathrm{H}_{2} \mathrm{O} 1097 \mathrm{GHz}$ lines with similar beam sizes (19") was considered to avoid beam-filling problems. The line-wing intensities

4 http://www.strw.leidenuniv.nl/ moldata/ 


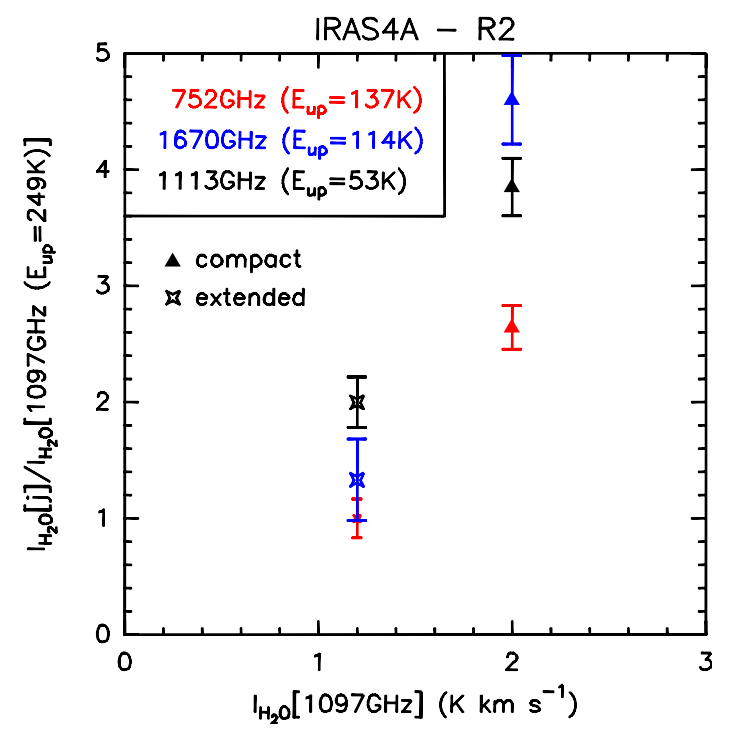

Fig. 9. $\mathrm{H}_{2} \mathrm{O}$ line ratios with respect to the o- $\mathrm{H}_{2} \mathrm{O}\left(3_{12}-3_{03}\right)$ line at $1097 \mathrm{GHz}$ measured at the R2 shock position, corresponding to the compact component, and at the offset position $\left(10^{\prime \prime},-10^{\prime \prime}\right)$ with respect to R2, corresponding to the extended component. The HIFI water line intensities are integrated in the line wings (see Fig. 10) at the original angular resolution of the spectra (see Table 2), while the intensity of the $\mathrm{H}_{2} \mathrm{O}$ line at $1670 \mathrm{GHz}$ in the two gas components is measured from the PACS map by averaging over $19^{\prime \prime}$. The intensity of the $\mathrm{H}_{2} \mathrm{O}\left(2_{11}-2_{02}\right)$ line at $752 \mathrm{GHz}$ associated with the compact component is corrected for the beam size ratio with respect to the $1097 \mathrm{GHz}$ line $\left(28^{\prime \prime} / 19^{\prime \prime}\right)$, assuming the source to be point-like.

(between $10 \mathrm{~km} \mathrm{~s}^{-1}$ and $60 \mathrm{~km} \mathrm{~s}^{-1}$ ) were measured at the same chosen positions corresponding to the two gas components. A comparison between the observed and predicted water line ratios as a function of the $\mathrm{H}_{2}$ density for four values of temperature $(100 \mathrm{~K}, 300 \mathrm{~K}, 500 \mathrm{~K}$, and $1000 \mathrm{~K})$ is presented in Fig. 10. In both cases, the low-density regime can be excluded, meaning that a gas density $n \gtrsim 10^{5} \mathrm{~cm}^{-3}$ is required. Such high densities are consistent with HIFI CS $(12-11)$ observations ${ }^{5}$ at a similar position about $\left(9^{\prime \prime}, 4^{\prime \prime}\right)$ offset from R1 (Gómez-Ruiz et al., in prep.), suggesting that gas densities in excess of $10^{5} \mathrm{~cm}^{-3}$ are needed to reproduce the CS (12-11) intensity in the line wing. They are also consistent with JCMT observations of broad CS (10-9) emission at the position of the IRAS 4A central source, probing warm and dense gas (Jørgensen et al. 2005).

To investigate the excitation conditions of the two $\mathrm{H}_{2} \mathrm{O}$ components at R2 in detail, we assumed that these components correspond to the warm and hot components found in previous works, that is, the warm component at about $300-500 \mathrm{~K}$ and the hot component at about $1000 \mathrm{~K}$ (see Santangelo et al. 2013; Busquet et al. 2014). By assuming these temperatures and modelling the observed $\mathrm{H}_{2} \mathrm{O} 1113 / 1097 \mathrm{GHz}$ line ratio and the intensity of the $\mathrm{H}_{2} \mathrm{O} 1097 \mathrm{GHz}$ line using the radex LVG code, we find that the hot component is associated with small emitting sizes (about $3^{\prime \prime}$, corresponding to about $700 \mathrm{AU}$ ), gas densities $n_{\mathrm{H}_{2}} \sim(1-4) \times 10^{5} \mathrm{~cm}^{-3}$, and $\mathrm{o}-\mathrm{H}_{2} \mathrm{O}$ column densities $\sim(0.5-1) \times 10^{16} \mathrm{~cm}^{-2}$ (corresponding to $\tau_{557 \mathrm{GHz}} \sim 15-40$ at the peak of the $\mathrm{H}_{2} \mathrm{O}$ emission). The warm component is instead more extended (sizes of about $10^{\prime \prime}-17^{\prime \prime}$, corresponding to 2400-4000 AU) and associated with higher gas densities $n_{\mathrm{H}_{2}} \sim(3-5) \times 10^{7} \mathrm{~cm}^{-3}$ and lower o- $\mathrm{H}_{2} \mathrm{O}$ column densities

\footnotetext{
5 The data are part of the OT1 program "Peering into the protostellar shocks: $\mathrm{NH}_{3}$ emission at high-velocities".
}

$\sim(1-2) \times 10^{13} \mathrm{~cm}^{-2}$ (corresponding to optically thin $\mathrm{H}_{2} \mathrm{O}$ emission, with $\left.\tau_{557 \mathrm{GHz}} \sim 0.04\right)$. The derived sizes for the extended and compact components are consistent with the fact that we cannot spatially resolve and separate them in the $\mathrm{H}_{2} \mathrm{O}$ emission with angular resolutions higher than 19" (see Fig. 7), while they can be spatially distinguished in the $\mathrm{CO}(3-2)$ emission, where the angular resolution is higher (14", see Fig. 8).

Next, we used the CO (16-15) emission observed at R2 to estimate the $\mathrm{H}_{2} \mathrm{O}$ abundance of the two spatial components. Since similar line profiles are observed for $\mathrm{CO}(16-15)$ and $\mathrm{H}_{2} \mathrm{O}$ at this position (see Sect. 3 and Fig. 2), we can assume that they share a common origin, meaning that the excitation conditions of $\mathrm{CO}(16-15)$ are the same as derived for $\mathrm{H}_{2} \mathrm{O}$ in both components. Since the CO (16-15) spectrum at R2 is a single-pointing observation, however, we cannot spatially separate the emission from the two components as we did with $\mathrm{H}_{2} \mathrm{O}$. Although the two components are not kinematically distinct (see Sect. 3.1), a crude way of separating their relative contribution to the intensity of the single observed $\mathrm{CO}(16-15)$ spectrum and thus deriving rough estimates of the $\mathrm{H}_{2} \mathrm{O}$ abundance, is to separate them in velocity; hence, we attribute the velocity range from $+11 \mathrm{~km} \mathrm{~s}^{-1}$ to $+30 \mathrm{~km} \mathrm{~s}^{-1}$ to the extended component and from $30 \mathrm{~km} \mathrm{~s}^{-1}$ to $45 \mathrm{~km} \mathrm{~s}^{-1}$ to the compact one, as suggested by the velocityintegrated maps of $\mathrm{CO}$ (3-2) (Fig. 8) and the CO (16-15) line profile. Assuming for each component the same excitation conditions and emission sizes as derived from $\mathrm{H}_{2} \mathrm{O}$, we obtain $\mathrm{CO}$ column densities of about $2 \times 10^{15} \mathrm{~cm}^{-2}$ and $2 \times 10^{16} \mathrm{~cm}^{-2}$ for the extended and compact components, respectively, which correspond to an $\mathrm{H}_{2} \mathrm{O} / \mathrm{H}_{2}$ abundance of about $(7-10) \times 10^{-7}$ for the warm extended component and (3-7) $\times 10^{-5}$ for the hot compact component (assuming a typical $\mathrm{CO} / \mathrm{H}_{2}$ abundance of $10^{-4}$ ). The low fractional $\mathrm{H}_{2} \mathrm{O}$ abundance associated with the warm gas component agrees with other studies of molecular outflows (e.g., Bjerkeli et al. 2012; Vasta et al. 2012; Nisini et al. 2013; Santangelo et al. 2013; Tafalla et al. 2013; Busquet et al. 2014). Moreover, our finding of higher fractional $\mathrm{H}_{2} \mathrm{O}$ abundance in the hot gas is consistent with ISO data (e.g., Giannini et al. 2001) and previous Herschel observations of shocked gas along the L1448 and L1157 outflows (e.g., Santangelo et al. 2013; Busquet et al. 2014).

We speculate that the compact hot component, detected in the $\mathrm{H}_{2} \mathrm{O}$ emission at $\mathrm{R} 2$, may be associated with the jet that impacts the surrounding material. Conversely, the warm, dense, and extended component originates from the compression of the ambient gas by the propagating flow. This picture was recently proposed by Busquet et al. (2014) for the L1157 outflow. Our data, however, allow for the first time to spatially resolve these two gas components through emission maps and confirm this scenario. We point out that high-angular resolution observations are crucial to probe the structure of the investigated shock region in depth.

\section{Conclusions}

We performed Herschel-HIFI observations of two shock positions (R1 and R2) along the IRAS 4A outflow. An area corresponding to the size of the largest HIFI beam of $38^{\prime \prime}$ at $557 \mathrm{GHz}$ was mapped in several key water lines with different upper level energies to study the water spatial distribution and to separate spatially different gas components associated with the shock. The main results of the work can be summarized as follows:

1. At both selected shock positions, we detect four $\mathrm{H}_{2} \mathrm{O}$ lines with upper energy levels in the range 50-250 K and 

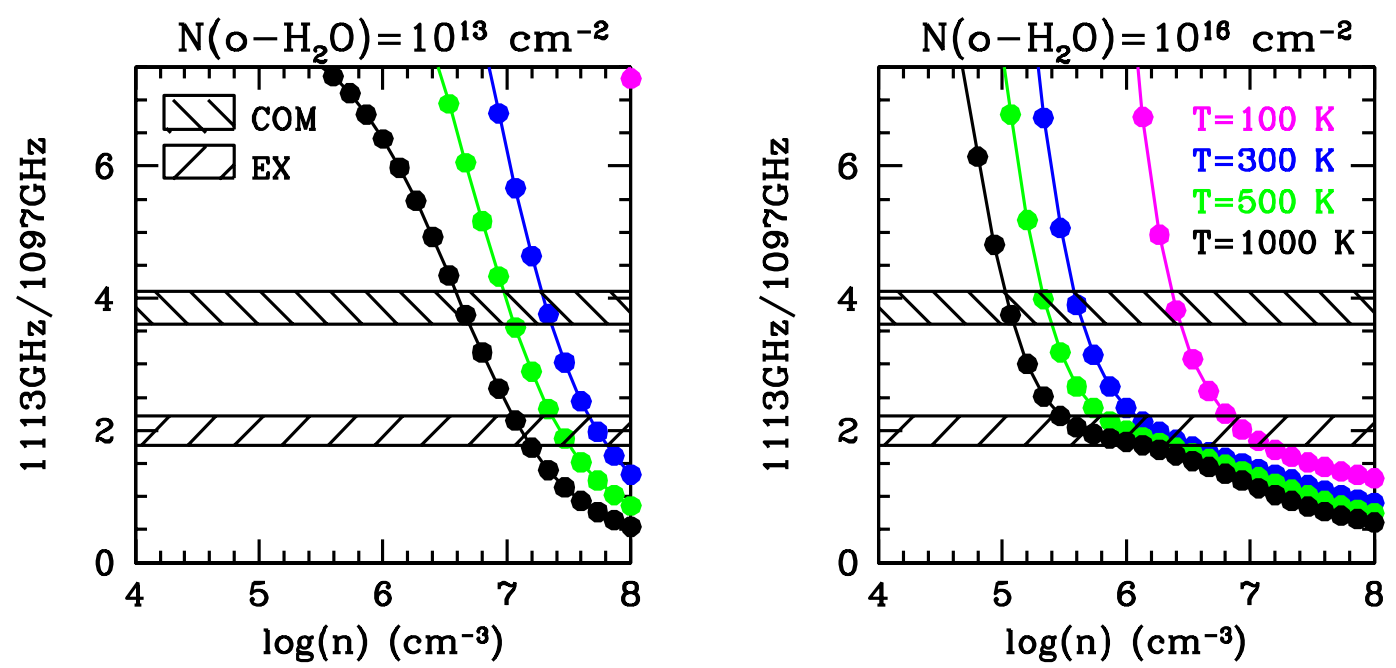

Fig. 10. Radex predictions for the ratio between the $\mathrm{p}-\mathrm{H}_{2} \mathrm{O}\left(1_{11}-0_{00}\right)($ at $1113 \mathrm{GHz})$ and the $\mathrm{o}-\mathrm{H}_{2} \mathrm{O}\left(3_{12}-3_{03}\right)$ (at $\left.1097 \mathrm{GHz}\right)$ lines as a function of the $\mathrm{H}_{2}$ density for four values of kinetic temperature $(100,300,500$, and $1000 \mathrm{~K})$ and two values of o- $\mathrm{H}_{2} \mathrm{O}$ column density $\left(10^{13}\right.$ and $\left.10^{16} \mathrm{~cm}^{-2}\right)$. The shaded bands represent the water ratios observed for the compact and extended components at R2, i.e., the R2 position and a position (10", $-10^{\prime \prime}$ ) offset from R2. The HIFI water line intensities are integrated in the line wings, between 10 and $60 \mathrm{~km} \mathrm{~s}^{-1}$, at the original angular resolution of the spectra $\left(19^{\prime \prime}\right)$.

Table A.1. Parameters of additional lines observed with HIFI.

\begin{tabular}{|c|c|c|c|c|c|c|c|c|c|c|}
\hline Line & Observation ID & Band & Mode $^{a}$ & Sideband & $\eta_{\mathrm{MB}}$ & $\begin{array}{r}\text { Frequency } \\
(\mathrm{MHz})\end{array}$ & $\begin{array}{r}E_{\mathrm{u}} / k_{\mathrm{B}} \\
(\mathrm{K})\end{array}$ & $\begin{array}{c}H P B W \\
(\operatorname{arcsec})\end{array}$ & $\begin{array}{l}\mathrm{R} 1 \\
(\mathrm{x}=\mathrm{y}\end{array}$ & $\begin{array}{l}\mathrm{R} 2 \\
\text { yes) }\end{array}$ \\
\hline $\mathrm{N}_{2} \mathrm{H}^{+}(6-5)$ & 1342248895,1342248897 & 1 & SP & LSB & 0.75 & 558966.50 & 94 & 38 & $\mathrm{x}$ & - \\
\hline $\mathrm{SO}\left(13_{14}-12_{13}\right)$ & 1342248895,1342248897 & 1 & SP & LSB & 0.75 & 560178.65 & 193 & 38 & $\mathrm{x}$ & - \\
\hline $\mathrm{CH}_{3} \mathrm{OH}\left(3_{-2}-2_{-1}\right)$ & 1342248895,1342248897 & 1 & SP & USB & 0.75 & 568566.05 & 40 & 38 & $\mathrm{x}$ & - \\
\hline $\mathrm{NH}_{3}\left(1_{0}-0_{0}\right)$ & 1342248895,1342248897 & 1 & SP & USB & 0.75 & 572498.16 & 27 & 37 & $\mathrm{x}$ & - \\
\hline${ }^{13} \mathrm{CO}(10-9)$ & 1342249853,1342249854 & 4 & M & LSB & 0.74 & 1101349.66 & 291 & 19 & $\mathrm{x}$ & $\mathrm{x}$ \\
\hline
\end{tabular}

Notes. ${ }^{(a)} \mathrm{SP}=$ Single Pointing mode; $\mathrm{M}=$ Mapping mode.

CO (16-15). In addition, transitions from related outflow and envelope tracers are detected.

2. At the R2 shock position, the head of the red-lobe of the outflow, two gas components with different excitation conditions are detected from the HIFI maps: a compact component, detected in the ground-state water lines, and a more extended component. They are not kinematically distinct, since the velocity range of the emission is similar in both cases, thus they cannot be distinguished within the large Herschel beam sizes of $19^{\prime \prime}$ and $38^{\prime \prime}$ at the frequencies of the groundstate $\mathrm{H}_{2} \mathrm{O}$ transitions.

3. The LVG analysis of the $\mathrm{H}_{2} \mathrm{O}$ emission suggests that the compact (about $3^{\prime \prime}$ ) component is associated with a hot $(T \sim 1000 \mathrm{~K})$ gas with densities $n_{\mathrm{H}_{2}} \sim(1-4) \times 10^{5} \mathrm{~cm}^{-3}$, while the extended $\left(10^{\prime \prime}-17^{\prime \prime}\right)$ component traces a warm $(T \sim 300-500 \mathrm{~K})$ and dense $\left(n_{\mathrm{H}_{2}} \sim(3-5) \times 10^{7} \mathrm{~cm}^{-3}\right)$ gas.

4. From a crude comparison between $\mathrm{H}_{2} \mathrm{O}$ and $\mathrm{CO}(16-15)$ emission observed at $\mathrm{R} 2$, we estimate the $\mathrm{H}_{2} \mathrm{O} / \mathrm{H}_{2}$ abundance of the warm and hot components to be $(7-10) \times 10^{-7}$ and $(3-7) \times 10^{-5}$.

Our $\mathrm{H}_{2} \mathrm{O}$ emission maps allow us, for the first time, to spatially resolve these two temperature components that were previously observed with HIFI and PACS. We suggest that the compact hot component is associated with the jet that impacts the surrounding material, while the warm, dense, and extended one originates from the compression of ambient gas by the propagating flow.

Acknowledgements. Herschel activities at INAF are financially supported by the ASI project 01/005/11/0. HIFI has been designed and built by a consortium of institutes and university departments from across Europe, Canada and the United States under the leadership of SRON Netherlands Institute for Space Research, Groningen, The Netherlands and with major contributions from Germany, France and the US. Consortium members are: Canada: CSA, U.Waterloo; France: CESR, LAB, LERMA, IRAM; Germany: KOSMA, MPIfR, MPS; Ireland: NUI Maynooth; Italy: ASI, IFSI-INAF, Osservatorio Astrofisico di Arcetri- INAF; The Netherlands: SRON, TUD; Poland: CAMK, CBK; Spain: Observatorio Astronómico Nacional (IGN), Centro de Astrobiología (CSIC-INTA); Sweden: Chalmers University of Technology - MC2, RSS \& GARD, Onsala Space Observatory, Swedish National Space Board, Stockholm University - Stockholm Observatory; Switzerland: ETH Zurich, FHNW; USA: Caltech, JPL, NHSC.

\section{Appendix A: Additional lines observed with HIFI}

The spectra of additional lines detected within the HIFI bands are presented in Fig. A.1 and summarized in Table A.1. We note that the R1 shock position seems to be more chemically rich than $\mathrm{R} 2$; more molecules are detected at R1 than at R2. The profiles of the lines detected at R1 show a red-wing component associated with the outflow and a low-velocity emission component possibly associated with the chemically enriched envelope material. The latter is clearly associated with the detection of $\mathrm{N}_{2} \mathrm{H}^{+}$and 
G. Santangelo et al.: Water distribution in shocked regions of NGC 1333-IRAS 4A
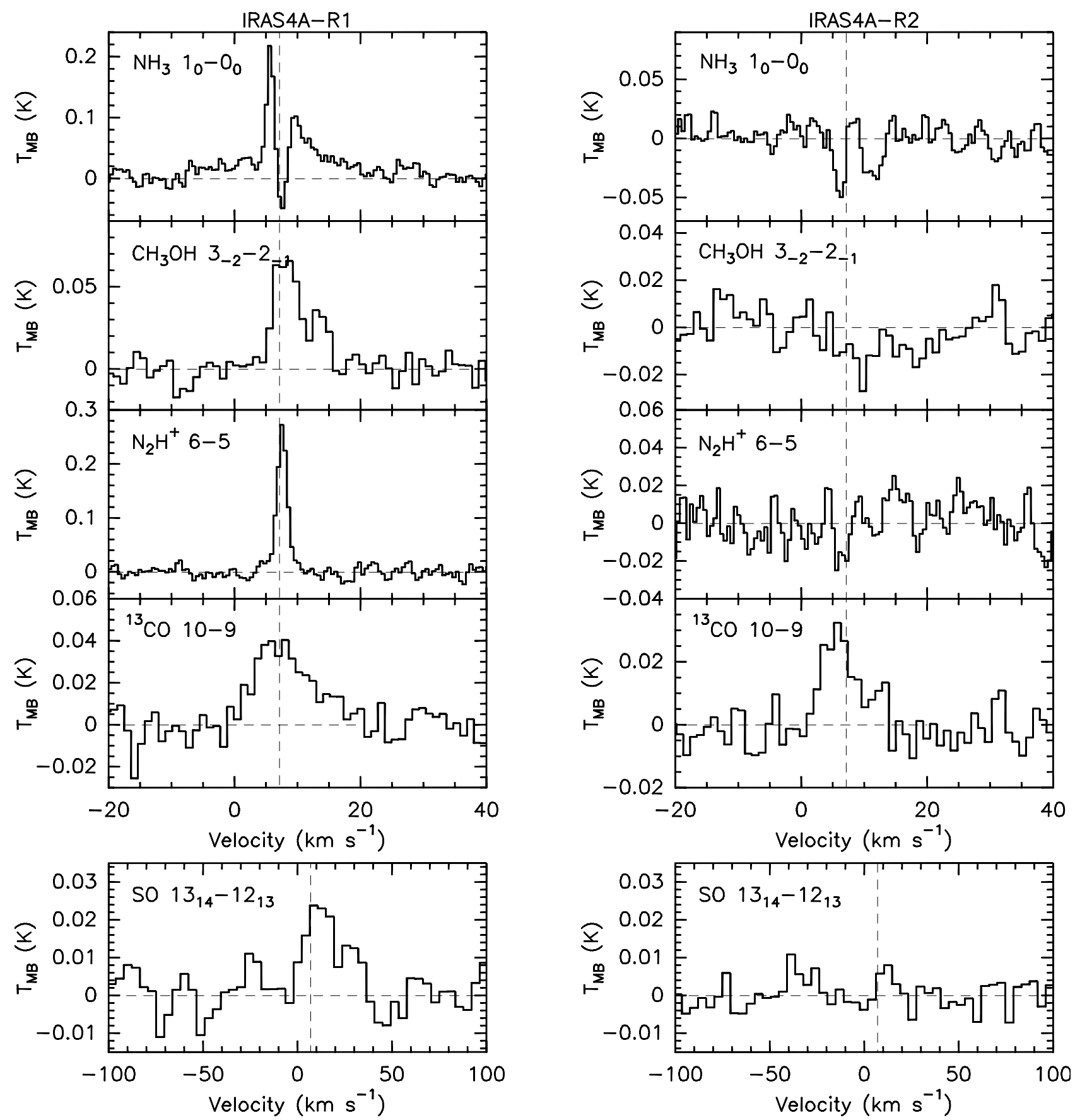

Fig. A.1. Additional lines observed with HIFI at the selected shock positions. All observations were taken in single-pointing mode, with the exception of the ${ }^{13} \mathrm{CO}(10-9)$ line. The absorption features seen in the $\mathrm{NH}_{3}$ spectrum at $\mathrm{R} 2$ are due to contamination from emission in the off-source reference position.

the absorption dip, centred at the source velocity, present in the $\mathrm{NH}_{3}$ spectrum. This detection is consistent with the beam size of the observations being large enough (about $38^{\prime \prime}$ ) to encompass part of the emission associated with the central driving source of the outflow, along with the emission coming from the outflow itself. We finally point out that the absorption features seen in the $\mathrm{NH}_{3}$ spectrum at $\mathrm{R} 2$ are due to contamination from emission in the off-source reference position.

\section{References}

Arce, H. G., Shepherd, D., Gueth, F., et al. 2007, in Protostars and Planets V (University of Arizona Press), 245
Baek, C. H., Kim, J., \& Choi, M. 2009, ApJ, 690, 944

Bally, J., Devine, D., \& Reipurth, B. 1996, ApJ, 473, L49

Bjerkeli, P., Liseau, R., Larsson, B., et al. 2012, A\&A, 546, A29

Blake, G. A., Sandell, G., van Dishoeck, E. F., et al. 1995, ApJ, 441, 689

Busquet, G., Lefloch, B., Benedettini, M., et al. 2014, A\&A, 561, A120

Choi, M. 2001, ApJ, 553, 219

Choi, M. 2005, ApJ, 630, 976

Choi, M., Kang, M., Tatematsu, K., Lee, J.-E., \& Park, G. 2011, PASJ, 63, 1281

Daniel, F., Dubernet, M.-L., Pacaud, F., \& Grosjean, A. 2010, A\&A, 517, A13

Daniel, F., Dubernet, M.-L., \& Grosjean, A. 2011, A\&A, 536, A76

de Graauw, T., Helmich, F. P., Phillips, T. G. et al. 2010, A\&A, 518, L6

Di Francesco, J., Myers, P. C., Wilner, D. J., Ohashi, N., \& Mardones, D. 2001, ApJ, 562, 770

Dionatos, O., Jørgensen, J. K., Green, J. D., et al. 2013, A\&A, 558, A88

Dubernet, M.-L., Daniel, F., Grosjean, A., et al. 2006, A\&A, 460, 323 
Dubernet, M.-L., Daniel, F., Grosjean, A., \& Lin, C. Y. 2009, A\&A, 497, 911 Emprechtinger, M., Lis, D. C., Rolffs, R., et al. 2013, ApJ, 765, 61 Flower, D. R., \& Pineau des Forêts, G. 2010, MNRAS, 406, 1745 Giannini, T., Nisini, B., \& Lorenzetti, D. 2001, ApJ, 555, 40 Girart, J. M., Crutcher, R. M., \& Rao, R. 1999, ApJ, 525, L109 Goicoechea, J. R., Cernicharo, J., Karska, A., et al. 2012, A\&A, 548, A77 Green, J. D., Evans, N. J., II, Jørgensen, J. K., et al. 2013, ApJ, 770, 123 Herczeg, G. J., Karska, A., Bruderer, S., et al. 2012, A\&A, 540, A84 Hirota, T., Bushimata, T., Choi, Y. K., et al. 2008, PASJ, 60, 37 Hollenbach, D., \& McKee, C. F. 1989, ApJ, 342, 306 Jørgensen, J. K., Schöier, F. L., \& van Dishoeck, E. F. 2005, A\&A, 437, 501 Jørgensen, J. K., Bourke, T. L., Myers, P. C., et al. 2007, ApJ, 659, 479 Karska, A., Herczeg, G. J., van Dishoeck, E. F., et al. 2013, A\&A, 552, A141 Kaufman, M. J., \& Neufeld, D. A. 1996, ApJ, 456, 611

Knee, L. B. G., \& Sandell, G. 2000, A\&A, 361, 671

Kristensen, L. E., Visser, R., van Dishoeck, E. F., et al. 2010, A\&A, 521, L30 Kristensen, L. E., van Dishoeck, E. F., Bergin, E. A., et al. 2012, A\&A, 542, A8 Kristensen, L. E., van Dishoeck, E. F., Benz, A. O., et al. 2013, A\&A, 557, A23 Lay, O. P., Carlstrom, J. E., \& Hills, R. E. 1995, ApJ, 452, L73

Liseau, R., Sandell, G., \& Knee, L. B. G. 1988, A\&A, 192, 153 Looney, L. W., Mundy, L. G., \& Welch, W. J. 2000, ApJ, 529, 477 Looney, L. W., Tobin, J. J., \& Kwon, W. 2007, ApJ, 670, L131

Manoj, P., Watson, D. M., Neufeld, D. A., et al. 2013, ApJ, 763, 83

Maret, S., Ceccarelli, C., Tielens, A. G. G. M., et al. 2005, A\&A, 442, 527
Maret, S., Bergin, E. A., Neufeld, D. A., et al. 2009, ApJ, 698, 1244 Nisini, B., Benedettini, M., Codella, C., et al. 2010, A\&A, 518, L120 Nisini, B., Santangelo, G., Antoniucci, S., et al. 2013, A\&A, 549, A16 Ott, S. 2010, in Astronomical Data Analysis Software and Systems XIX, ASP Conf. Ser. 434, 139

Pilbratt, G. L., Riedinger, J. R., Passvogel, T., et al. 2010, A\&A, 518, L1

Ray, T., Dougados, C., Bacciotti, F., Eislöffel, J., \& Chrysostomou, A. 2007, Protostars and Planets V (Tucson: University of Arizona Press), 231

Roelfsema, P. R., Helmich, F. P., Teyssier, D., et al. 2012, A\&A, 537, A17

Sandell, G., Aspin, C., Duncan, W. D., Russell, A. P. G., \& Robson, E. I. 1991, ApJ, 376, L17

Santangelo, G., Nisini, B., Giannini, T., et al. 2012, A\&A, 538, A45

Santangelo, G., Nisini, B., Antoniucci, S., et al. 2013, A\&A, 557, A22

Schöier, F. L., van der Tak, F. F. S., van Dishoeck, E. F., \& Black, J. H. 2005, A\&A, 432, 369

Suutarinen, A. N., Kristensen, L. E., Mottram, J. C., Fraser, H. J., \& van Dishoeck, E. F. 2014, MNRAS, 440, 1844

Tafalla, M., Liseau, R., Nisini, B., et al. 2013, A\&A, 551, A116

van der Tak, F. F. S., Black, J. H., Schöier, F. L., Jansen, D. J., \& van Dishoeck, E. F. 2007, A\&A, 468, 627

van Dishoeck, E. F., Kristensen, L. E., Benz, A. O., et al. 2011, PASP, 123, 138 Vasta, M., Codella, C., Lorenzani, A., et al. 2012, A\&A, 537, A98

Yildız, U. A., Kristensen, L. E., van Dishoeck, E. F., et al. 2012, A\&A, 542, A86

Yıldı, U. A., Kristensen, L. E., van Dishoeck, E. F., et al. 2013, A\&A, 556, A89 